\title{
Semiconductors as Studied by Magnetic Resonance Methods
}

\author{
Pavel G. Baranov $\cdot$ H. Jürgen von Bardeleben
}

Published online: 9 June 2010

(C) Springer-Verlag 2010

Being honored to edit a special issue of Applied Magnetic Resonance we were highly inspired by the possibility to emphasize the role of magnetic resonance techniques for the investigation of semiconductors. In recent years, magnetic resonance studies have undergone a noticeable shift towards the characterization of different organic systems, where also very fruitful approaches towards the development of new magnetic resonance techniques were elaborated. In an attempt to present these developments, we have decided to highlight the most significant results obtained in semiconductor materials and nanostructures studies as in particular they are one of the most crucial phase of the development of new technologies and applications.

This special issue includes many comprehensive reviews that represent state-ofthe-art approaches towards the study of various semiconductors, such as zinc oxide, silicon carbide and silicon, boron nitride, gallium arsenide and others. The papers that follow highlight the importance of magnetic resonance methods, such as electron paramagnetic resonance (EPR), optically detected magnetic resonance, electron-nuclear double resonance and others for the investigation of various intrinsic and impurity-related defects and dopants which are of exceptional potential for technological applications. Moreover, a notable contribution to the development of the EPR technique that lay well beyond the usual experimental framework is reported in one of these exceptional papers.

It is hoped that this special issue on advances in magnetic resonance studies in semiconductors will be in resonance with further investigations and developments.

P. G. Baranov ( $ه)$

Ioffe Physical-Technical Institute, Polytekhnicheskaya 26, 194021 St. Petersburg, Russia

e-mail: pavel.baranov@mail.ioffe.ru 
We express our gratitude to the authors who have contributed to this special issue. We are also grateful to Prof. K. Salikhov and Dr. L. Mosina. Without their efforts, the publication of this special issue would not have been possible. 